\title{
Robotized system for retrieving fallen objects within the reactor vessel of a nuclear power plant (PWR).
}

\author{
$\therefore$ Iborra, B. Alvarez,P.J. Navarro, J.M. Fernández \\ Universidad Politécnica de Cartagena \\ Electronics Technology Department \\ Campus Muralla del Mar, s/n, 30202 Cartagena, Spain \\ Andres.Iborra@upct.es
}

\author{
J.A. Pastor-Franco \\ Enwesa Operaciones, S.A. \\ R\&D Department \\ Avda. Juan Carlos I, 8, 39600 Maliaño, Cantabria, Spain \\ japastor@enwesa.es
}

\begin{abstract}
This paper presents an original teleoperated and robotized system (TRON) designed for retrieving foreign objects within lower internals of the reactor vessels at nuclear power plants (PWR). For performing these operations, the system does not require that the lower internals have to be retrieved or the fuel assemblies unloaded. The remote handler device is an articulated pole for accessing to the lower internals from the lower core plate. This cylindrical pole is capable to reach the $90 \%$ of the horizontal surface below the core barrel. In order to carry out such mission, the TRON system must be able to underwater operate inside a very complex environment and exposed to moderate levels of radiation, that could be punctually high or very high. The design and implementation of the teleoperation system is based on generic software architecture. This original architectural model has been a very useful scheme for organising TRON control tasks, since it comprises the main functional subsystems that may appear in a teleoperation system and clearly defines the interconnections among them. The use of a generic architecture for the system development has allowed guaranteed its success. The system has been tested in one of the nuclear power plants where it will work (C.N. Asco).
\end{abstract}

\section{INTRODUCTION.}

In PWR nuclear power stations the refuelling is carried out once every two years. In the execution of these maintenance tasks exist the possibility that fall objects inside the reactor vessel due to human errors or other circumstances. In [1] this problem has been studied, one of these incidents not takes place foreseen at least every 40.000 hours of the reactor operation.

The retrieving operations of fallen objects inside the reactor vessels are usually very delicate due to the characteristics of the environment where such activities are performed. These maintenance tasks have to be performed underwater at a depth of 10 to 20 meters, with levels of radiation usually moderate, but that could be in occasions very high. The working environment is complex and full of obstacles, the access paths are very narrow and the visibility is difficult due to the multiple barriers to light.

In the last years the number of teleoperated and robotized systems for working inside the reactor's vessel, such as robots, arms and different kinds of poles, is significantly increased and improved, providing nuclear plants with new and reliable technologies for vessel's maintenance. Some examples of this are, among others, the URSULA ${ }^{\mathrm{TM}}$ robot of Framatome [2], the PIV ${ }^{\mathrm{TM}}$ system of Tecnatom [3]; and some versions of the ROSA ${ }^{T M}$ arm of Westinghouse [4].
Most of these systems are designed for exhaustive and previously scheduled inspections of the vessel's parts, which usually require the unloading of the fuel assemblies and the removal of the internals. The systems are usually large, heavy and hard to install, but the time and resources employed on it are not significant respect to the total operation time and the benefits that its use implies. However, and due to the same reasons, they are not very appropriate for-punctual and not previously scheduled operations, such as the retrieval of foreign objects accidentally fallen into the internals.

The proposed system has been designed for retrieving foreign objects from the inside of the reactor vessels of the PWR nuclear plants and for inspections of small areas under the lower core plate. The main design requirement was that the system could be used without removing the lower internals nor a significant number of fuel assemblies, and that the installation of the system in the working areas could be performed by a reduced number of operators in a short period of time.

\section{WORKING ENVIRONMENT.}

The reactor vessel (figure 1) is cylindrical with a hemispherical bottom head and flanged removable upper head. The vessel contains the core, the core support structures, control rod clusters, neutron shield panels and other parts directly associated with the core. The vessel's dimensions vary from one plant to another, but the overall length of assembled vessel, closure head and nozzles is usually about 13 meters and the inside diameter of the shell is about 4 meters. The distance from the vessel top, excluding the upper head, to the water surface is around 10 meters. The core is supported within the reactor vessel by the lower internals. The main components of these are the core barrel, the lower core support plate, the core baffle, the lower instrumentation guide tubes and the antisismic plates. The lower core support structure (mainly the core barrel) also serves to provide passageways and control for the coolant flow through the core.

Under these working conditions, foreign objects may come to rest on some of the horizontal surfaces of the reactor, such as on the top of fuel assemblies, the core baffle top or the lower core plate. In these cases the objects are generally visible by underwater cameras and can be retrieved by experts operators using suction devices, pincers or similar equipment. 

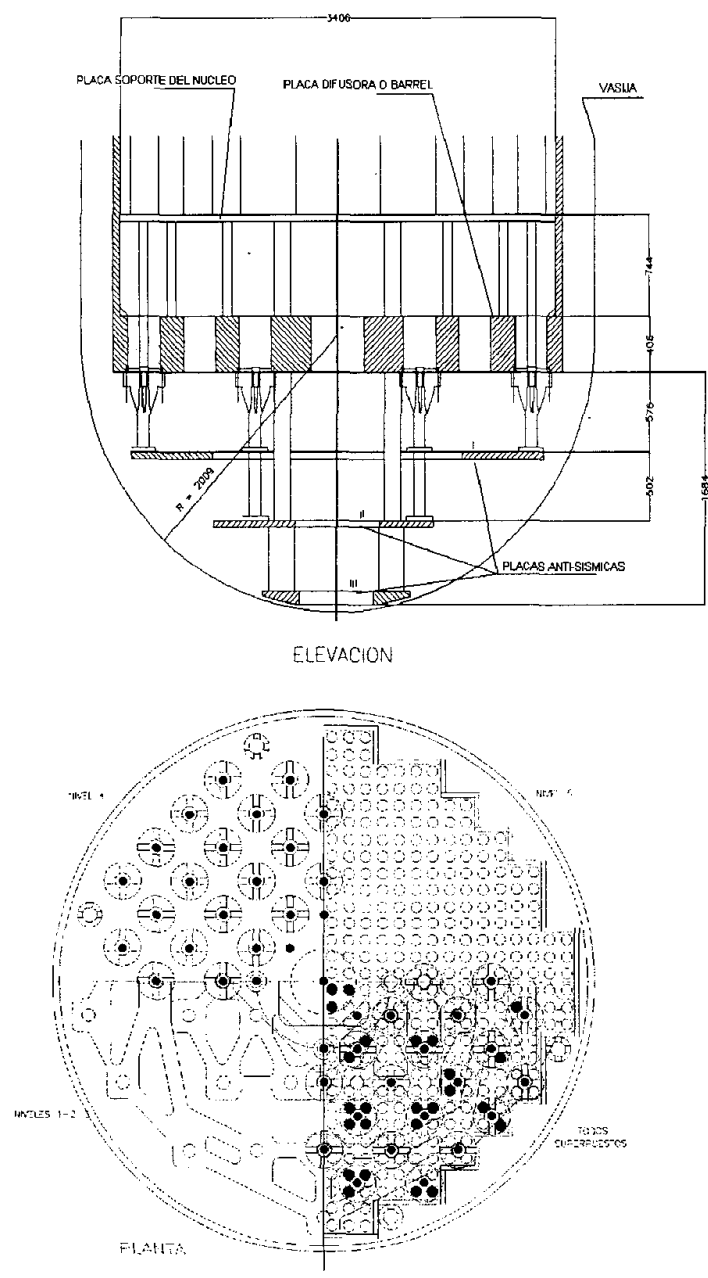

Fig. 1. PWR reactor vessel.

However, it is possible that foreign objects pass through one of the holes of the lower core plate and they come to rest on the core support plate or on some of the tie plates underneath, or even fall down all the way to the bottom of the reactor vessel. In these cases the objects are not visible by conventional means, its source and characteristics may be unknown as well as its actual location, and it may represent a severe threat to the fuel or to other reactor parts if left inside the vessel.

Another mechanism whereby foreign objects may end up at the bottom of the reactor vessel is by being carried by the coolant flow from other parts of the reactor coolant system, such as the steam generators or the coolant pumps, where they may have been accidentally introduced in the course of inspection and maintenance operations.

Foreign objects represent an unacceptable threat to the core or other parts of the reactor and should be retrieved. The retrieval of these objects by conventional means from the lower part of the reactor requires unloading completely the core, transferring all the fuel assemblies to the fuel building and then remove the entire reactor internals from the vessel and store them underwater in the reactor cavity. Once the vessel is completely empty, the inspection and retrieval can be performed using long handle underwater cameras and conventional tools. However, the removal of the reactor internals is a time consuming operation. For it, this operation is not normally done in the course of regular refuelling operations.

TRON system performances this operation without retrieving the lower internals nor unloading a significant number of fuel assemblies ( 1 to 4$)$. The system is capable of performing the following missions:

- Visual inspection of the structures under the lower core plate.

- Retrieval of foreign objects from inside the lower internals.

According the working environment described, it was defined the system requirements presented in the next section.

\section{SYSTEM REQUIREMENTS.}

In order to performance the above missions, the following system requirements were defined for TRON:

- TRON system should be capable to operate between 15 and 20 meters underwater and resist moderate levels of radiation that could be punctually high or very high.

- It should be capable to pass through the holes of the lower core plate (about $65 \mathrm{~mm}$ of diameter).

- The mechanical devices should be enough robust in order to resist small collisions, without damage or loss of pieces moving, at the maximum speed.

- It is mandatory that the mechanical devices could be removed from the internals under any circumstance, including mechanical, electrical or software failure or loss of power.

- The system should aid the operator to reach the objects avoiding the obstacles (most of them the columns under the lower core plate and the barrel).

It is also was taking into consideration that the mentioned missions above would not usually be previously scheduled and then the system should be easy to transport, install and set-up. For the above reasons, it was established that:

- The weight of the devices to be installed inside the vessel could not exceed $30 \mathrm{Kg}$.

- The system could be dismantled into small parts that could be transported and assembled by one or two operators at the proximity of the reactor vessel.

- The system should be loaded and unloaded from the fuel crane's platform or from the edge of the reactor pit and these tasks should not required the used of heavy or huge auxiliary structures.

Additionally, a very strong requirement that it have been took in account during the design phase was that the TRON system was as modular as possible, and the used 
components (hardware and software) were commercial ones and not ad-hoc solutions. The objective was to develop a low cost system which the components could be easily replaced in the case it was necessary.

\section{MECHANICAL SUPPORT.}

The figure 2 shows the developed mechanical support of the TRON system. This consists of the following parts:

- A remote handler device consisting of an articulated pole for accessing the lower internals from the lower core plate. This pole is capable to reach the $90 \%$ of the horizontal surfaces below the core barrel.

- The end effectors, which fall into two categories: (1) a set of tools, pneumatic and electric, for retrieving foreign objects, including pincers, grippers, suction devices and magnets; and (2) a set of inspecting sensors that, like the tools, are attached at the end of the pole. These tools are attached to the end of the pole and are provided with cameras or sensors.

- A visualization system based on computer vision algorithms, capable to distinguish the main components of the lower internals and a variety of shapes. This system provides assistance to the operator matching real live images of the environment with the associated virtual images.

- The control system. This subsystem receives the commands from the operator and decides actions activating robot and tool actuators. In general it supervise and execute the operator orders.

\section{The remote handler device.}

The remote handler device is a very slight cylindrical pole that comprises four joints moves by DC motors (figure 4). The pole is divided into assemblies and its main features are:

- Length: Between $\mathbf{1 5 0 0}$ and $\mathbf{4 5 0 0} \mathrm{mm}$ depending on the number of parts assembled.

- Weight: $28 \mathrm{Kg}, 18$ of the pole and 10 of the base for attaching it to the lower core plate.

- Load Capacity: $1.4 \mathrm{Kg}$ if joint 3 is mounted, $10 \mathrm{Kg}$ working in vertical position.

- Storage and transport: None of the assemblies, except the base, is longer than 1 meter. So it is transported and stored inside two relatively small boxes (one for the assemblies and one for the base) that can be carried by an operator.

- Watertighness: The pole can work 20 meters underwater. The motors are inside watertigh boxes that are pressurized with dry air in order to prevent water to damage the motors.

- Radiation resistant: There are not electronic components inside the pole. The motor position is read from resolvers, since the encoders can be damaged by radiation.

- Materials: The materials used for the pool have been titanium and stainless steel. Aluminium has been used for those parts that are not in contact with vessel parts. None of the materials used can interact with the water reactor. Titanium have been used in order to reduce the pool weight at maximum.

The pole base: The pole is attached to the lower core plate by means this module. It also provides to the pole the necessary rigidity and includes the motorization and gears for joint 1 . The design of the base depends on the specific geometry of each lower core plate. It takes up the surface of just one fuel assembly. The base currently implemented corresponds to the lower internals of the nuclear plant of Ascó $I$ in Spain. It is provided with two pins and a pneumatic cam-lock that fix it to the plate. The camlock can be manually release from the fuel crane in case of pneumatic failure.

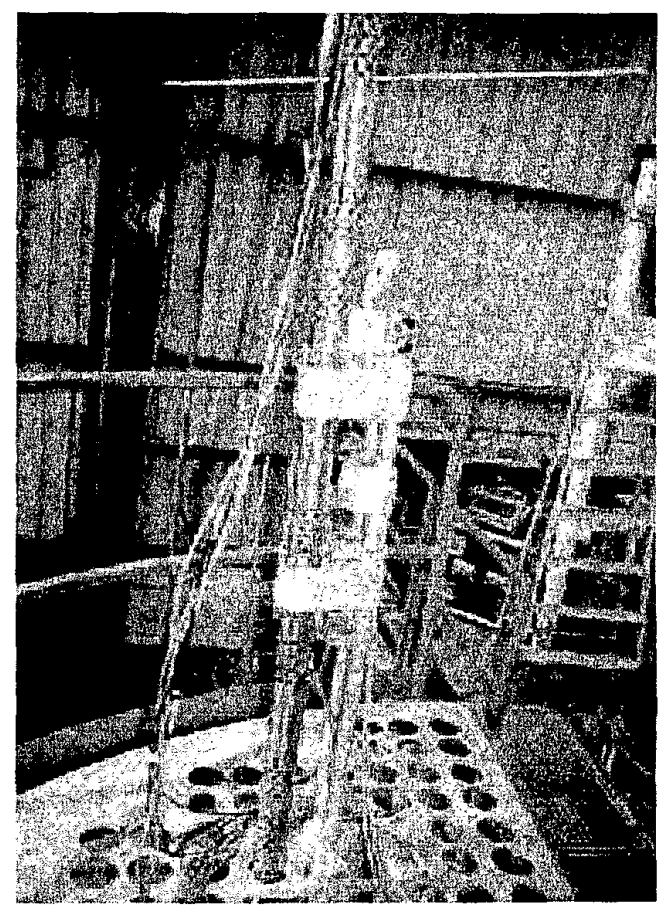

Fig. 2. Mechanical support.

\section{End effectors.}

The end effectors fall into two categories: Those design for retrieving foreign objects, such as pneumatic and electric, for retrieving foreign objects, including pincers, grippers, suction devices and magnets and those specifically designed for inspection tasks. Though the TRON system is designed for these operations it could perform other tasks if they don't require the use of heavy tools (load capacity is limited to $1.4 \mathrm{~kg}$ if joint 3 is used).

All the tools are provided with a quick connector for its attachment at the end of the pole that comprises all the required connections, power supply (electric, pneumatic or hydraulic), control (reception of commands and transmission of status information) and video. For this reason the load and unload of the tools is an easy and fast operation. 


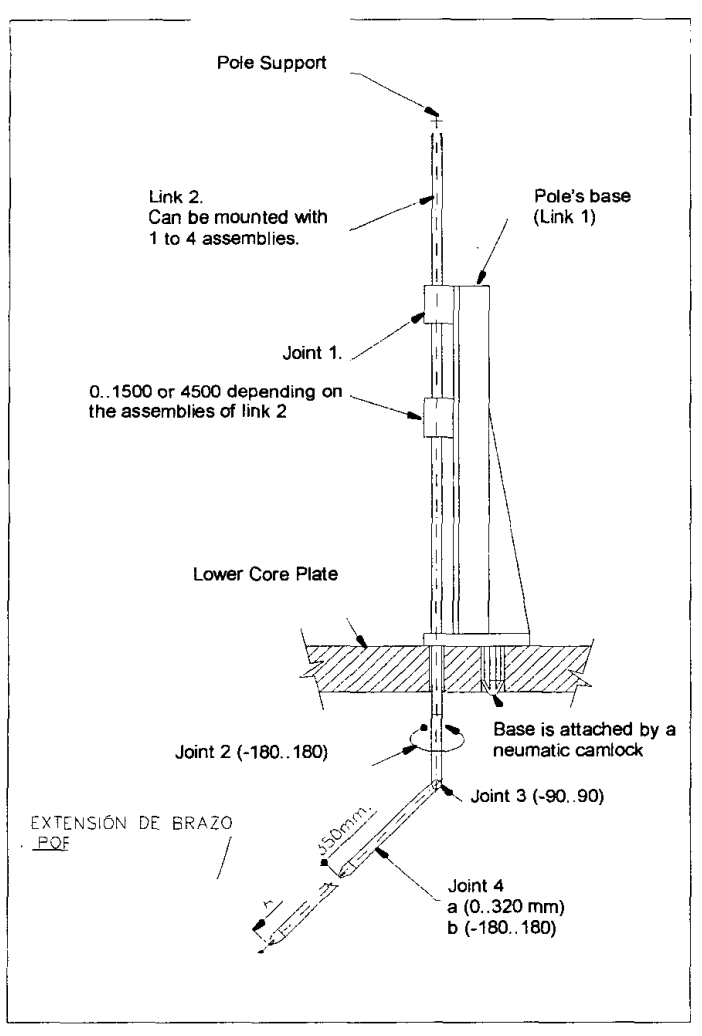

Fig. 3. Schematic view of the pole

The tools also include, at least, one camera and one light. The end effector's cameras are the unique component of TRON that can be damaged by radiation, but the worsening of its functioning is gradual and doesn't imply risk. The possibility of use radiation-resistant cameras is not realistic due its size and weight. However, in order to allow a long visual inspection of the internals, a specific end effector's has been designed to support one compact radiation-resistant camera. One of the tools (a pincer) is provided with a fiberscope. Some fiberscopes resist the radiation and may be a good solution for those applications where the required depth of observation and field of view are not very large (about $300 \mathrm{~mm}$ and $45^{\circ}$ respectively).

\section{GLOBAL ARCHITECTURE.}

Figure 4 shows a scheme of global architecture for the control system of the pole and tools. Two subsystems can be identified. The first one, explained in the next section, is a teleoperation platform based on the use of a personal computer, and the other is a non intelligent control unit (control boxes). Teleoperation platform provides a great versatility to the system and offers to operator a great amount of information about the devices and the environment allowing a very precise control over them. The control boxes are included for control in case of application failure. They also can be used for simple tasks where a precise positioning of the pole was not required. The switching between one method of control to the other doesn't require changes in the connections, it is enough to change the position of a switch in the amplifiers box. It is required for safety reasons that control boxes are always available when using the application. However, the control boxes can be used alone.

\section{TELEOPERATION PLATFORM.}

The approach taken for teleoperation platform is based on the generic architecture described in [4][5]. Such architecture is shown in figure 5. According to [4][5] the pole and the end-effectors have been described in terms of their basic commands, their states machines and their structural and dynamic models.

Devices characterisation

- Basic commands:

- Pole:

- Controller configuration: Selection of the environment (vessel), pole (TRON model), end-effector, mode of operation and TRON base location.

- Enable/Disable joint.

- Motion commands. Only joint motion commands are defined and among them, motions involving just a joint are the most common. This is due to the space constrains (see figures 1, 2 and 4) imposed by the environment. The attempts to execute a linear trajectory, or simply to execute a motion involving two or more joints, used to cause a collision.

- End-effectors:

- Controller configuration: The end-effectors currently used have just a mode of operation, so there are not configuration commands defined for them. Tool controllers are loaded during pole configuration and all the required initialisations are automatically performed.

- Enable/Disable tool.

- Activate/Deactivate Tool.

- Motion commands. There aren't motion commands defined for the end-effectors. Though some of them have a joint, this is added to pole during its configuration.

- State Machines: They define the operational state of the pole and end-effectors.

- Structural description and dynamic models of pole and end-effectors: Only used for simulation purposes and off-line programming.

Generic architecture applied in this work also considers composite commands (set of basic commands executed sequentially) and automatic commands (operations that are executed automatically when an specific condition hold). The only composite commands defined are those referring to pole installation and the unique automatic command is to stop and disable the joints when a collision is detected. 


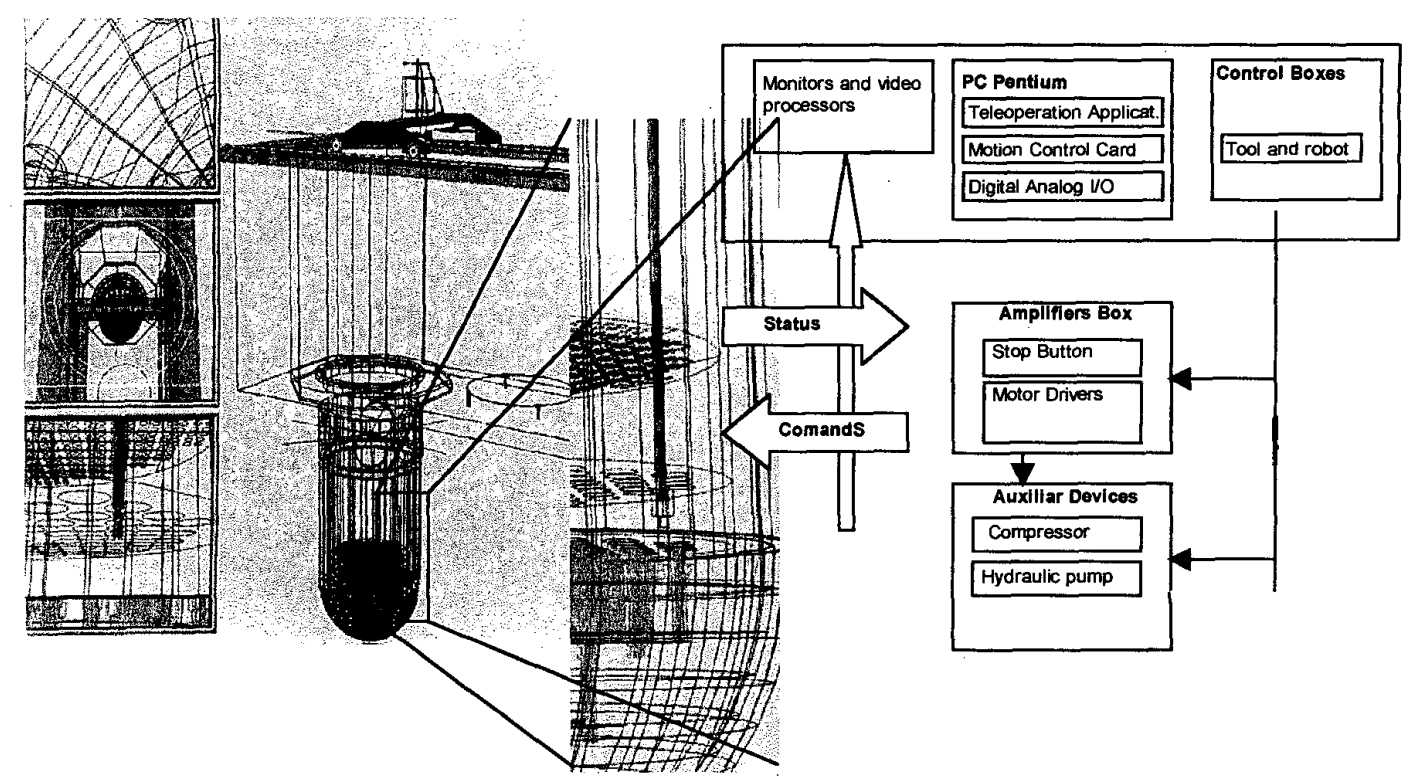

Fig. 4. Global architecture.

\section{Architecture instantiation}

Controllers. There are two controllers, one corresponding to the pole and the other corresponding to the end effector, which represent their respective states of execution. Controllers' functioning is based on a previous feasibility check of the commands sent by the operator and on the continuos monitorisation of commands' execution and device's status. A command is feasible if it is allowed in the current mode of operation and will leave the system in a known and safe state. For example, any attempt of operator to execute a motion command while pole is not safely attached to vessel's internals will be rejected, since motion commands are not allowed during pole installation (installation mode). In the same way, a motion cormmand that may cause a collision will not be allowed though it is allowed attending to the current operation mode. It is important to notice that controllers are not in charge of direct sensing and actuation. They translate the user commands to the sequence of actions that device has to execute and send this sequence, through the communication module, to lower level processes, that execute them. In the opposite way, controllers process the information coming from lower level processes and according to it decide the subsequent actions to be performed.

Controllers received the commands from user interface. The rest of the systems acts like servers that are invoked by the controllers in order to get the information required for feasibility check and system monitorisation.

Collision detection queries are used only for feasibility check of the commands, but not for monitorisation purposes due to timing reasons. During motion, the controller does not send queries to the kinematic-graphic server and the collisions are detected reading the input current of the motors.
When a motion command is issued, the kinematic server calculates a set of intermediate points that the pole have to reach at a given instants (this is performed before starting the motion). While motion is executing the controller compares the expected position with the real position. If the difference is within a previously defined tolerance the motion continues, else the motion is aborted. When the controller sends a motion command to pole, it simultaneously requests graphical server that executes the same motion. That is, the real motion and its simulation run in parallel. The operator should realise that the graphical representation of the motion is only a simulation and only corresponds to reality if the motion is executing like expected.

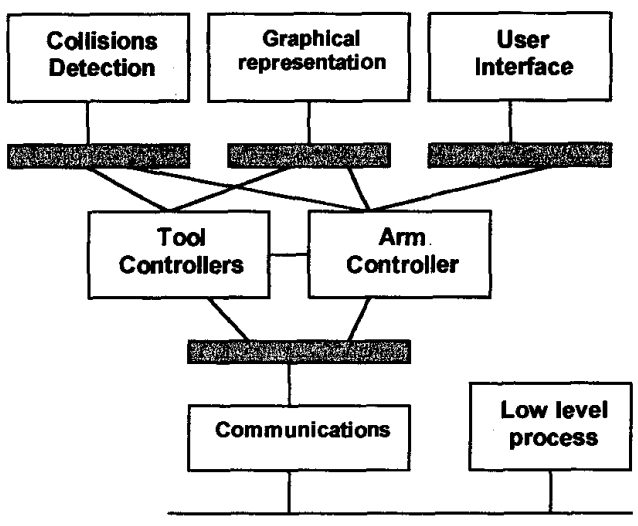

Fig. 5. Generic architecture for teleoperation.

User Interface. The user interface provides operator with access to all pole's and end-effector's commands and shows him the current system status, including a schematic view of the working environment. The operation interface 
is set after devices configuration, since commands depends on the current pole model and end-effector attached.

kinematic\&Graphic server. This server provides controller and operator with kinematic \& graphical functions, including collision detection and motion simulation. This module is used for feasibility check of motion commands (collision detection), for simulation purposes (off-line programming) and for monitoring purposes (generation of expected path). Though the architecture defined in [4][5] separates the kinematic and graphical issues, commercial tools offered both services together, so we prefer to consider both in the same module.

Communication module. This module represents the link between the controllers and the lower level processes those in charge of direct sensing and actuation. The architecture assumes that controller processes and lower level process may be distributed in different platforms. This is not the case for TRON. These processes run in the same platform and communication is performed by means of critical sections.

Implementation details.

The controllers, the low level processes and the user interface run in the same platform, a Pentium PC, and the programmed in Microsoft Visual $\mathrm{CH}$. The operating system used has been Windows NT. Each one of these elements is a thread process. Comunications among them are performed by means of critical sections. The graphical\&kinematic server runs on a workstation HP 725, and it's based on the utilities provided by ROBCAD. Communications links between processes running in the $\mathrm{PC}$ and the graphical\&kinematic server are performed by means of TCP/IP sockets. The controllers have been design and implemented to be reused. A generic mechanism controller class has been defined at the top of the hierarchy class. The pole and end-effector controllers have been derived from such mechanism controller class. The objectoriented programming paradigms allow designing software general enough to be adapted or extended if new functionality has to be added.

Due to the slow speed of the pole a real-time operating system has not been required. The most catastrophic situation that may happens is a collision, but at the current motion speeds the pole is not damaged. When pole collides with environment, the motor torque increases very quickly. This variation can be detected monitoring the motor input current, and motor can be disable before it results damaged.

\section{CONCLUSIONS AND FUTURE WORKS.}

The generic architectural model [4][5] has been a very useful scheme for organising the TRON control tasks, since it comprises the main functional subsystems that may. appear in a teleoperation system and clearly defines the interconnections among them.
The use of a generic architecture for development of the system has allowed guaranteed its exit. The software has been tested successfully, with the real robot and a 1:1 mock-up of the nuclear plant part where it will work.

The $\mathrm{C}++$ language paradigms fit very well with the architectural approach and software modules have been developed with relatively little effort.

In future versions the navigation system, that currently works alone, will be integrated with the rest of the system providing controller with additional information about the environment status. Finally, future versions will have to consider the use of a real time operating system, since its lack make impossible the use of the current software with faster and/or weaker devices.

\section{ACKNOWLEDGEMENTS}

This work has been partially supported by the Spanish Government Programmes for Research and Development ( ATYCA 163/96, ATYCA 110/97, ATYCA 107/98 and CDTI, EUREKA_MAINE TRON EU 1565)

\section{REFERENCES.}

[1] Toribio, E - Non Schedule Incidents in PWR Nuclear Power Plants- Proceedings of the $20^{\text {th }}$ Annual Meeting of the Spanish Nuclear Society (SNE), Cordoba 1994, Spain.

[2] Glass, S.W., and Fairbrother, D.B., 1995 AURSULA A compact walking robot for Reactor Vessel examination $5^{\text {th }}$ International Conference for Robotics in Hazardous Environments, Palo Alto, C.A.

[3] J.L. Sánchez et al 1997.- Proyecto PIV (Penetraciones de Instrumentación del fondo de vasijas de centrales PWR).- $23^{\text {rd }}$ Annual Meeting of the Spanish Nuclear Society (SNE), La Coruña 1997.

[4] Al. Alonso, B. Alvarez, J.A. Pastor, J.A. de la Puente and A.lborra. "Software architecture for a robot teleoperation system" $4^{\text {th }}$ IFAC Workshop on Algorithms and Architectures for Real-time Control, April 1997.

[5] B. Alvarez, A.lborra, A.Alonso, J.A de la Puente. "Reference architecture for robot teleoperation: Development details and practice use". Proceeding of the $6^{\text {th }}$ IFAC Workshop on Algorithms and Architectures for Real-Time Control, May 2000.

[6] B. Álvarez, A. Alonso, and J.A. de la Puente. "Timing Analysis of a generic robot teleoperation software architecture", Control Engineering Practice, Vol.6, no.6, pag. 409-416, June, 1998.

[7] B.Alvarez, A.lborra, A.Alonso, J.A. de la Puente, J.A. Pastor. "Developing multi-application remote systems". Nuclear Engineering International, Vol. 45, no. 548. March 2000. 